\title{
State of Power Estimation of Echelon-Use Battery Based on Adaptive Dual Extended Kalman Filter
}

\author{
Enguang Hou ${ }^{1,2}{ }^{\oplus}$, Yanliang $X u^{1, *}$, Xin Qiao $^{2}$, Guangmin Liu $^{2}$ and Zhixue Wang ${ }^{2}$ \\ 1 School of Electrical Engineering, Shandong University, Jinan 250061, China; houenguang@mail.sdu.edu.cn \\ 2 School of Rail Transportation, Shandong Jiao Tong University, Jinan 250357, China; \\ 215043@sdjtu.edu.cn (X.Q.); 215040@sdjtu.edu.cn (G.L.); 215035@sdjtu.edu.cn (Z.W.) \\ * Correspondence: xuyanliang@sdu.edu.cn
}

Citation: Hou, E.; Xu, Y.; Qiao, X.; Liu, G.; Wang, Z. State of Power Estimation of Echelon-Use Battery Based on Adaptive Dual Extended Kalman Filter. Energies 2021, 14, 5579. https://doi.org/10.3390/en14175579

Academic Editor: Gianfranco Chicco

Received: 5 August 2021

Accepted: 3 September 2021

Published: 6 September 2021

Publisher's Note: MDPI stays neutral with regard to jurisdictional claims in published maps and institutional affiliations.

Copyright: (c) 2021 by the authors. Licensee MDPI, Basel, Switzerland. This article is an open access article distributed under the terms and conditions of the Creative Commons Attribution (CC BY) license (https:// creativecommons.org/licenses/by/ $4.0 /)$.

\begin{abstract}
Owing to the degradation of the performance of a retired battery and the unclear initial value of the state of charge (SOC), the estimation of the state of power (SOP) of an echelon-use battery is not accurate. An SOP estimation method based on an adaptive dual extended Kalman filter (ADEKF) is proposed. First, the second-order Thevenin equivalent model of the echelon-use battery is established. Second, the battery parameters are estimated by the ADEKF: (a) the SOC is estimated based on an adaptive extended Kalman filtering algorithm, that uses the process noise covariance $Q_{k}$ and observes the noise covariance $R_{k}$, and (b) the ohmic internal resistance and actual capacity are estimated based on the aforementioned algorithm, that uses the process noise covariance $Q_{\theta, k}$ and observes the noise covariance $R_{\theta, k}$. Third, the working voltage and internal resistance are predicted using optimal estimation, and the SOP of the echelon-use battery is estimated. MATLAB simulation results show that, regardless of whether or not the initial value of the SOC is clear, the proposed algorithm can be adjusted to the adaptive algorithm, and if the estimation accuracy error of the echelon-use battery SOP is less than $4.8 \%$, it has high accuracy. This paper provides a valuable reference for the prediction of the SOP of an echelon-use battery, and will be helpful for understanding the behavior of retired batteries for further discharge and use.
\end{abstract}

Keywords: echelon-use battery; adaptive dual extended Kalman filter; state of power; second-order Thevenin equivalent model

\section{Introduction}

In recent years, with the increase of the number of retired batteries, the cascade utilization of power batteries has attracted increasingly more attention. The echelon-use battery refers to the lithium iron phosphate powered lithium battery used in EV (electric vehicle) when the capacity attenuates to less than $80 \%$, and more than $20 \%$, which is used for the power backup and energy storage of the communication base station. There are many works in the literature devoted to the second use of retired EV batteries. Back in 2010, Neubauer et al. [1] believed that the second use strategies had the potential to become a common part of the future automotive battery life cycle. Tong et al. [2] and Omar et al. [3] verified the feasibility of the retired vehicle batteries used in an off-grid photovoltaic vehicle charging system and clarified the capacity decay trend of cells. The result showed that the testing cells possessed another 1400 or 1000 cycles of life while they were charged/discharged at $1 \mathrm{C}$ and $80 \%$ depth of discharge (DOD). Jiang et al. [4] estimated the second-life battery remaining capacity by three types of regression methods and concluded that the correlation-based feature selection method was feasible and the estimation error was within 3\%. Schuster et al. [5] assessed the correlation between the capacity and impedance of lithium-ion cells during calendar life as a base for capacity quick tests and found that the $\mathrm{SOH}$ (state of health) quick test must be parameterized with aging data close to actual use. YL et al. [6] tested the performance of retired EV battery modules in order to learn their attenuation states, and different capacity test protocols of retired 
modules were compared in order to strike a balance between calibration accuracy and test time. When the vehicle battery was retired, the capacity was below $80 \%$, the retired cells possessed another more than 1000 cycles of life while they were charged/discharged at $1 \mathrm{C}$ and $80 \%$ depth of discharge (DOD) [2,3]. The retired modules still have a good discharge ability, implying that a retired battery energy storage system can be employed to satisfy the power demand of an electricity grid.

Because of the performance degradation and capacity attenuation of a ladder battery, its safety is difficult to guarantee, leading to the difficulty of large-scale promotion and utilization of cascade batteries. The state of power (SOP), as an important parameter for battery safety control and energy recovery, has attracted significantly greater attention. The battery experimental method and model method are commonly used to estimate SOP. The battery experimental method is a test method adopted by the United States Advanced Battery Consortium (USABC) [7] that has the advantage of strong practicability [8]. Fang [9] of Central South University, China, and Yu [10] of Harbin University of Science and Technology, China, adopted the experimental method. The disadvantage of this method is that the testing is complicated, and equipment is required. Therefore, the use of the estimation method based on the equivalent circuit model has increased. Plett [11] was the first to propose the method of estimating peak power based on the equivalent circuit model. In $[12,13]$, the first-order equivalent circuit model is used by the authors to estimate the SOP. In [14-19], the second-order Thevenin equivalent model is used by the authors to estimate SOP, and an accuracy of $7.2 \%$ is obtained.

Using the model method to estimate the SOP has the advantages of simplicity and low equipment requirements. However, the estimation accuracy of the SOP by this method depends on the accuracy of the equivalent model of the echelon battery itself. Owing to the degradation of echelon-use battery performance, its equivalent model is far from meeting the estimation accuracy requirements of the SOP; therefore, an important research direction of battery SOP estimation is to improve the accuracy of the equivalent model of an echelon-use battery and the adaptive ability of the algorithm.

To sum up, the research object of this paper is the echelon-use battery. Most researchers did not consider the impact of battery performance attenuation on SOP estimation. In addition, due to performance attenuation, the estimation error of the SOC increases, and there is a large error between the SOC and the actual value when it is reused. Aiming at the above problems of echelon-use battery performance, an adaptive dual extended Kalman filter (ADEKF) algorithm is first applied based on the second-order Thevenin equivalent model. That is, based on the ADEKF algorithm used to estimate the SOC, ohmic internal resistance, and actual capacity, the optimal estimation is used to predict the working voltage and ohmic internal resistance to estimate the echelon-use battery SOP. Finally, the estimation method of the cascade battery SOP is established to provide a safe guarantee for the promotion and utilization of the cascade battery.

The objective of this study is to propose an effective SOP estimation method for echelon-use batteries and analyze the influence of the degradation of the performance of such batteries and the SOC estimation on the SOP estimation. Four original contributions are made herein.

(1) In this paper, the parameter estimation of the echelon-use battery is discussed. Compared with the new battery, the SOC initial value is not clear, and the parameters are degraded.

(2) In view of the unclear SOC, an adaptive method is adopted, that can be adjusted adaptively to estimate the accuracy based on process noise covariance $Q_{k}$ and observe the noise covariance $R_{k}$.

(3) To improve the accuracy of SOP estimation, an adaptive Kalman filter algorithm is adopted to estimate the parameters of echelon-use batteries in real time based on process noise covariance $Q_{\theta, k}$ and observing the noise covariance $R_{\theta, k}$, aiming at the performance attenuation of the echelon-use battery and the inaccuracy of actual capacity and ohmic internal resistance. 
(4) The impact of echelon-use battery SOC and working voltage on the SOP estimation is presented.

\section{The Second Order Thevenin Equivalent Model of Echelon-Use Battery}

A lithium battery is a very complex nonlinear system. It is necessary to choose a higher-order battery equivalent model to simulate the characteristics of the battery more accurately. The second-order Thevenin equivalent model can not only better reflect the dynamic and static characteristics of the battery but can also lower the order of the model, which reduces the calculation of the processor and makes it easy to implement in engineering applications [20].

In this paper, the second-order Thevenin model is used as the equivalent model of an echelon-use battery, and the equivalent circuit diagram is shown in Figure 1. Indeed, $\tau_{1}=R_{1} C_{1}$ is the short constant of time, which is used to simulate the process of rapid change of discharge voltage in the dynamic characteristics of the battery; $\tau_{2}=R_{2} C_{2}$ is the time length constant, which is used to simulate the process of slow and stable discharge voltage in the dynamic characteristics of the battery. Furthermore, $U_{o c}$ is the open circuit voltage of the battery, $U_{L}$ is the working voltage of the battery, $i_{L}$ is the charge and discharge current of the battery, $R_{0}$ is the ohmic internal resistance of the battery.

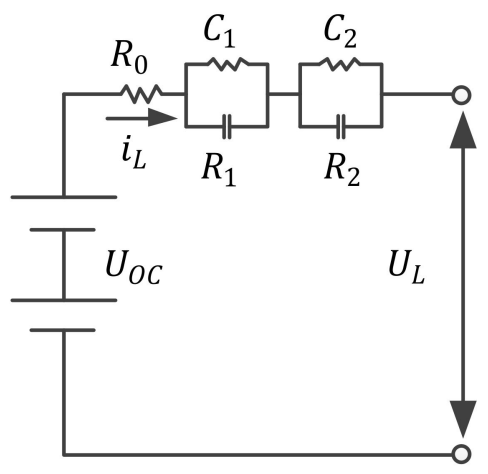

Figure 1. The second-order Thevenin equivalent model.

From Figure 1, the discrete state equation of the second-order equivalent circuit of the echelon-use cell is as follows:

$$
\left[\begin{array}{c}
S_{c k+1} \\
U_{k+1}^{R_{1} C_{1}} \\
U_{k+1}^{R C_{2}}
\end{array}\right]=\left[\begin{array}{ccc}
1 & 0 & 0 \\
0 & \exp \left(-\frac{\Delta t}{\tau_{1}}\right) & 0 \\
0 & 0 & \exp \left(-\frac{\Delta t}{\tau_{2}}\right)
\end{array}\right] \cdot\left[\begin{array}{c}
S_{c k} \\
U_{k}^{R_{1} C_{1}} \\
U_{k}^{R_{2} C_{2}}
\end{array}\right]+\left[\begin{array}{c}
-\frac{\Delta t}{C} \\
R_{1}\left(1-\exp \left(-\frac{\Delta t}{\tau_{1}}\right)\right) \\
R_{2}\left(1-\exp \left(-\frac{\Delta t}{\tau_{2}}\right)\right)
\end{array}\right] \cdot i_{k}+\omega_{k}
$$

From Figure 1, the discrete observation equation of the second-order equivalent circuit of the echelon-use cell is as follows:

$$
U_{k}=\left[\left.\frac{d\left(U_{o c}\left(S_{C}\right)\right)}{d S_{C}}\right|_{S_{C}=S_{C K}}-1-1\right] \cdot\left[\begin{array}{c}
S_{c k} \\
U_{k}^{R_{1} C_{1}} \\
U_{k}^{R_{2} C_{2}}
\end{array}\right]-i_{k} R_{0}+\vartheta_{k}
$$

As

$$
\begin{gathered}
A_{k}=\left[\begin{array}{ccc}
1 & 0 & 0 \\
0 & \exp \left(-\frac{\Delta t}{\tau_{1}}\right) & 0 \\
0 & 0 & \exp \left(-\frac{\Delta t}{\tau_{2}}\right)
\end{array}\right], B_{k}=\left[\begin{array}{c}
-\frac{\Delta t}{C} \\
R_{1}\left(1-\exp \left(-\frac{\Delta t}{\tau_{1}}\right)\right) \\
R_{2}\left(1-\exp \left(-\frac{\Delta t}{\tau_{2}}\right)\right)
\end{array}\right], x_{k}=\left[\begin{array}{c}
S_{c k} \\
U_{k}^{R_{1} C_{1}} \\
U_{k}^{R_{2} C_{2}}
\end{array}\right], \\
C_{k}=\left[\left.\frac{d\left(U_{o c}\left(S_{C}\right)\right)}{d S_{C}}\right|_{S_{C}=S_{C K}}-1-1\right], u_{k}=i_{k} .
\end{gathered}
$$

So

$$
f\left(x_{k}, u_{k}\right)=A_{k} x_{k}+B_{k} u_{k}
$$




$$
g\left(x_{k}, u_{k}\right)=C_{k} x_{k}-R_{0, k} u_{k}
$$

where, $S_{c k}, S_{c k+1}$ are the SOC of the cascade battery at $k, k+1$ time in the discrete state; $C$ is the actual capacity of the cascade battery, the unit is $A \cdot h ; i_{k}$ is the charge and discharge current at $k$ time in the discrete state; $U_{k}$ is the working voltage of the cascade battery at $\mathrm{k}$ time in the discrete state; $\Delta t$ is the sampling period; $U_{k}^{R_{1} C_{1}}, U_{k+1}^{R_{1} C_{1}}$ are the estimated voltage values of $R_{1}$ at $k, k+1$ time in the discrete state; $U_{k}^{R_{2} C_{2}}, U_{k+1}^{R_{2} C_{2}}$ are the estimated voltage values of $R_{2}$ at $k, k+1$ time in the discrete state; $\omega_{k}, \vartheta_{k}$ are independent system noises; $U_{o c}\left(S_{C}\right)$ is the open circuit voltage of the battery corresponding to the SOC value of the cascade battery at the $k$ time in the discrete state.

\section{Parameter Identification}

\subsection{Open Circuit Voltage}

The open circuit voltage $U_{O C}$ :

(1) Charge at the current of $30 \mathrm{~A}$ and stop at the cell voltage of $3.65 \mathrm{~V}$.

(2) Discharge at the current of $30 \mathrm{~A}$ and stop at the cell voltage of $2.5 \mathrm{~V}$. Record the discharge voltage $U_{d i s}$.

(3) Charge at the current of $30 \mathrm{~A}$ and stop at the cell voltage of $3.65 \mathrm{~V}$. Record the charge voltage $U_{c h}$.

(4) The open circuit voltage $U_{O C}$ is as follows:

$$
U_{O C}=\frac{U_{d i s}+U_{c h}}{2}
$$

\subsection{Ohmic Internal Resistance}

According to Ohm's law, the ohmic internal resistance $R_{0}$ is as follows:

$$
R_{0}=\frac{\Delta U}{\left|i_{\mathrm{L}}\right|}
$$

where $\Delta U$ is voltage change, $i_{L}$ is the charge and discharge current of the battery [20].

\subsection{Polarization Resistance}

The working voltage $U_{L}$, charge-discharge current $i_{L}$ and open circuit voltage $U_{O C}$ of the echelon-use battery obtained through the charge-discharge test. The least square method is used to minimize the sum of the squares of the residual and identify the parameter model. This method is introduced in detail in the literature [21], so it will not be repeated.

\section{SOP Estimation}

In this paper, an adaptive dual Kalman filter algorithm is applied based on the second-order Thevenin equivalent model: the first is to estimate the SOC based on the adaptive extended Kalman filter (AEKF) algorithm; the second is to estimate the ohmic internal resistance and actual capacity based on the adaptive extended Kalman filter (AEKF) algorithm [22,23].

\subsection{SOC Estimation Based on AEKF}

According to Equations (3) and (4), the variable of the echelon-use battery system is the SOC. In this paper, the ohmic resistance and actual capacity of the echelon-use battery are added into the state variable due to the serious problems of the increase of ohmic resistance and the decline of the actual capacity of the echelon-use battery. The system state variable has three parameters: SOC, ohm internal resistance, and the actual capacity $[24,25]$.

The state and observation equations are as follows:

$$
x_{k+1}=f\left(x_{k}, u_{k}, \theta_{k}\right)+\omega_{k}
$$




$$
y_{k+1}=g\left(x_{k}, u_{k}, \theta_{k}\right)+\vartheta_{k}
$$

where, $\theta_{k}$ is the state variable ohmic internal resistance and actual capacity, $\theta_{k}=\left[R_{0, k}, C_{k}\right] ; x_{k}$ is the system state variable; $u_{k}$ is the input of the system and is the cascade battery current; $y_{k}$ is the system observation variable and is the working voltage of the echelon-use battery.

In this paper, the error covariance matrices of the zero-mean Gaussian white noise $\omega_{k}$ and $\vartheta_{k}$ are $Q_{k}$ and $R_{k}$.

The algorithm flow is as follows:

- $\quad$ Step 1: Initialize $x$ is as follows:

$$
\begin{gathered}
\hat{x}_{0}=E\left(x_{0}\right) \\
\hat{P}_{0}=E\left[\left(x_{0}-\hat{x}_{0}\right)\left(x_{0}-\hat{x}_{0}\right)^{T}\right.
\end{gathered}
$$

- $\quad$ Step 2: Time update of the system state $x$ is as follows:

$$
\begin{gathered}
x_{k}=f\left(\hat{x}_{k-1}, u_{k-1}, \theta_{k}\right) \\
P_{k}=A_{k-1} \hat{P}_{k-1} A_{k-1}^{T}+Q_{k}
\end{gathered}
$$

- $\quad$ Step 3: Status update of the system state $x$ is as follows: The Kalman gain is as follows:

$$
K_{k}=P_{k} C_{k}^{T}\left(C_{k} P_{k} C_{k}^{T}+R_{k}\right)^{-1}
$$

The optimal estimation of state variables is as follows:

$$
\hat{x}_{k}=x_{k}+K_{k}\left[y_{k}-g\left(\hat{x}_{k-1}, u_{k-1}, \hat{\theta}_{k-1}\right)\right]
$$

The optimal estimate of the covariance is as follows:

$$
\hat{P}_{k}=\left(E-K_{k} C_{k}\right) P_{k}
$$

- Step 4: Process noise covariance equation is as follows:

$$
Q_{k}=\left(1-d_{k}\right) Q_{k-1}+d_{k}\left[K_{k}\left(\hat{y}_{k}-y_{k}\right)\left(\hat{y}_{k}-y_{k}\right)^{T} K_{k}^{T}+P_{k}-A_{k-1} \hat{P}_{k-1} A_{k-1}^{T}\right]
$$

- $\quad$ Step 5: Observe the noise covariance equation as follows:

$$
R_{k}=\left(1-d_{k}\right) R_{k-1}+d_{k}\left[\left(\hat{y}_{k}-y_{k}\right)\left(\hat{y}_{k}-y_{k}\right)^{T}-C_{k} P_{k} C_{k}^{T}\right]
$$

where $d_{k}=\frac{1-b}{1-b^{k}}, k=1,2, \cdots, n, b$ is the forgetting factor, $0<b<1 ; \hat{x}_{k}$ is the optimal state estimation value at $k$ time of sampling; $x_{k}$ is the estimated value of the state variable at $k$ time of sampling; $\hat{y}_{k}$ is the actual observed value at $k$ time of sampling; $y_{k}$ is the estimated value of the observed variable at $k$ time of sampling; $\hat{P}_{k}$ is the optimal estimation value of error covariance at $\mathrm{k}$ time of sampling; $P_{k}$ is the estimated value of error covariance at $k$ time of sampling.

\subsection{The Ohm Internal Resistance and Actual Capacity Estimation Based on AEKF}

The state and observation equations of the system with the newly added state parameters are as follows:

$$
\begin{gathered}
\theta_{k+1}=\theta_{k}+\gamma_{k} \\
D_{k+1}=g\left(x_{k}, u_{k}, \theta_{k}\right)+e_{k}
\end{gathered}
$$

where $\gamma_{k}$ is the noise on the input variable, $e_{k}$ is the noise on the output variable; an adaptive extended Kalman filter algorithm is applied to the state variable $\theta$ to obtain the real-time estimation results of the internal resistance and actual capacity of the battery. In order to obtain the accurate ohmic resistance and actual capacity of the battery, the error 
between the estimated value of the observation variable and the actual value of the work of the echelon battery was corrected [26,27].

In this paper, the error covariance matrices of the zero-mean Gaussian white noise $r_{k}$ and $e_{k}$ are $Q_{\theta, k}$ and $R_{\theta, k}$.

The algorithm flow is as follows:

- $\quad$ Step 1: Initialize $\theta$ as follows:

$$
\begin{gathered}
\hat{\theta}_{0}=E\left(\theta_{0}\right) \\
P_{\theta, 0}=E\left[\left(x_{0}-\hat{x}_{0}\right)\left(x_{0}-\hat{x}_{0}\right)^{T}\right]
\end{gathered}
$$

- Step 2: Time update of the system state $\theta$ is as follows:

$$
\begin{gathered}
\theta_{k}=\hat{\theta}_{k-1} \\
P_{\theta, k}=\hat{P}_{\theta, k-1}+Q_{\theta, k}
\end{gathered}
$$

- Step 3: Status update of the system state $\theta$ is as follows: The Kalman gain is as follows:

$$
K_{\theta, k}=P_{\theta, k} C_{k}^{T}\left(C_{k} P_{\theta, k} C_{k}^{T}+R_{\theta, k}\right)^{-1}
$$

The optimal estimation of state variables is as follows:

$$
\hat{\theta}_{k}=\theta_{k}+K_{\theta, k}\left[y_{k}-g\left(\hat{x}_{k-1}, u_{k-1}, \hat{\theta}_{k-1}\right)\right.
$$

The optimal estimate of the covariance is as follows:

$$
\hat{P}_{\theta, k}=\left(E-K_{\theta, k} C_{k}\right) P_{\theta, k}
$$

- Step 4: Process noise covariance equation is as follows:

$$
Q_{\theta, k}=\left(1-d_{\theta, k}\right) Q_{\theta, k-1}+d_{\theta, k}\left[K_{\theta, k}\left(\hat{y}_{k}-y_{k}\right)\left(\hat{y}_{k}-y_{k}\right)^{\mathrm{T}} K_{\theta, k}^{\mathrm{T}}+P_{\theta, k}-A_{k-1} \hat{P}_{\theta, k-1} A_{k-1}^{\mathrm{T}}\right]
$$

- Step 5: Observe the noise covariance equation as follows:

$$
R_{\theta, k}=\left(1-d_{\theta, k}\right) R_{\theta, k-1}+d_{\theta, k}\left[\left(\hat{y}_{k}-y_{k}\right)\left(\hat{y}_{k}-y_{k}\right)^{\mathrm{T}}-C_{k} P_{\theta, k} C_{k}^{\mathrm{T}}\right]
$$

where $d_{\theta, k}=\frac{1-b_{\theta}}{1-b_{\theta}^{k}}, k=1,2, \cdots, n, b_{\theta}$ is the forgetting factor, $0<b_{\theta}<1$.

\subsection{SOP Estimation Based on ADEKF}

The SOP optimal estimate forecast is updated as follows:

The optimal estimation of the predicted working voltage at time $k+1$ is as follows:

$$
\hat{U}_{k+1}=g\left(\hat{x}_{k}, u_{k}, \hat{\theta}_{k}\right)=\left[\left.\frac{d\left(U_{o c}\left(S_{C}\right)\right)}{d S_{C}}\right|_{S_{C}=S_{C K}}-1-1\right] \cdot\left[\begin{array}{c}
S_{c k} \\
U_{k}^{R C_{1}} \\
U_{k}^{R_{2} C_{2}}
\end{array}\right]-i_{k} R_{0, k}
$$

The optimal estimation prediction SOP at time $k+1$ is as follows:

$$
P_{k+1}=\frac{\hat{U}_{k+1}^{2}}{R_{0, k+1}+R_{1}+R_{2}}
$$

Ignore the effect of internal polarization resistance:

$$
P_{S O P}=\frac{\hat{U}_{k+1}^{2}}{R_{0, k+1}}
$$


where $g\left(\hat{x}_{k-1}, u_{k-1}, \hat{\theta}_{k-1}\right)$ is the estimated value of the observed variable at $k$ time of sampling.

\subsection{The Flow Chart of SOP Estimation Based on ADEKF}

The flow chart of the SOP estimation of an echelon-use battery is shown in Figure 2.

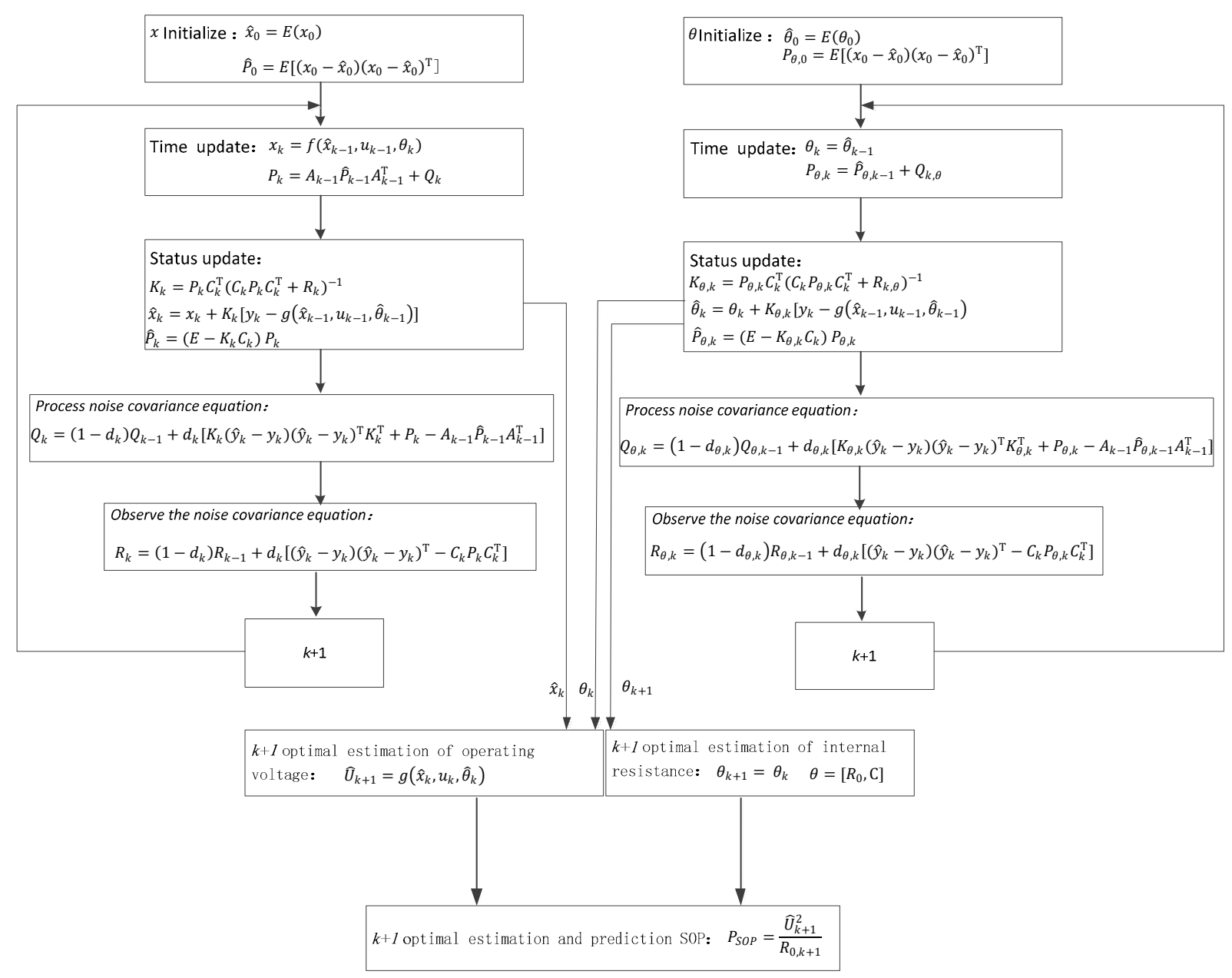

Figure 2. The flow chart of SOP estimation of echelon-use battery.

\section{Simulation and Discussion}

\subsection{SOC Estimation Based on AEKF}

In Figure 3, a lithium iron phosphate echelon-use battery (60 AH, $3.2 \mathrm{~V}$, Table 1) was selected to conduct the charge/discharge experiment using BTS20-5V/4*300A/WD (Hubei Techpow Electric Co., Ltd., China) monomer battery charging-discharging equipment at room temperature. The fully charged echelon battery was discharged several times to simulate the simple working conditions. Different discharge currents were used each time for simulation verification and analysis using MATLAB R2020a (MathWorks, Natick, USA).

To verify the adaptive characteristics of the algorithm, a discharge experiment was carried out on a fully charged echelon battery, starting from an SOC of $100 \%$ and ending at an SOC of $25 \%$. In the process of simulation verification, the initial SOC values were changed to $100 \%, 70 \%$, and $40 \%$ separately, and the adaptive and error curves were observed and analyzed.

In the simulation verification, the actual values of the SOC, working voltage, and SOP were acquired by the charging-discharging equipment (BTS20-5V/4*300A/WD), and the estimated values were calculated using the ADEKF algorithm. 


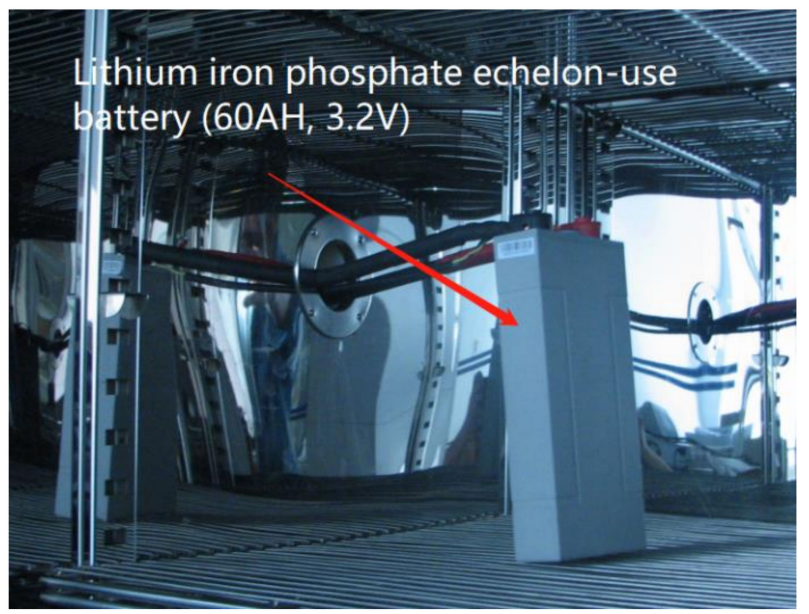

(a)

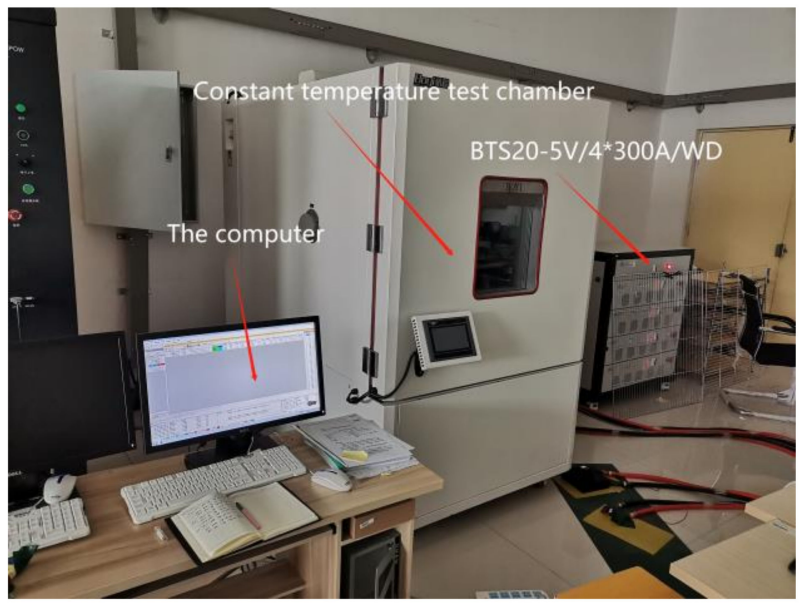

(b)

Figure 3. Experimental environment. (a) The lithium iron phosphate echelon-use battery (60 AH, 3.2 V). (b) The charge/discharge experiment.

Table 1. Parameters of the echelon-use battery.

\begin{tabular}{ccc}
\hline Items & Parameter & Remarks \\
\hline capacity & $60 \mathrm{Ah}$ & $60 \mathrm{~A}$ \\
nominal voltage & $3.2 \mathrm{~V}$ & \\
working voltage & $2.5 \mathrm{~V}$ to $3.65 \mathrm{~V}$ & $20 \mathrm{~A}$ \\
charging time & $3 \mathrm{~h}$ & \\
charging temperature & $0{ }^{\circ} \mathrm{C}$ to $45^{\circ} \mathrm{C}$ & \\
discharging temperature & $-20^{\circ} \mathrm{C}$ to $55^{\circ} \mathrm{C}$ & \\
\hline
\end{tabular}

The formula for error:

By formula (1), (2), (14), $\hat{x}_{k}=\left[\begin{array}{c}\hat{S}_{c k} \\ \hat{U}_{k}^{R_{1} C_{1}} \\ \hat{U}_{k}^{R_{2} C_{2}}\end{array}\right]$, the first behavior is the optimal value $\hat{S}_{C k}$ of SOC.

The SOC error equation is as follows:

$$
\text { SOC error }=\hat{S}_{C k}-S_{a c t u a l}
$$

where, $S_{\text {actual }}$ is the value collected by the device. is $\hat{U}_{k+1}$.

By formula (29), the optimal estimation of the predicted working voltage at time $k+1$

The working voltage error equation is as follows:

$$
\text { Working voltage error }=\hat{U}_{k+1}-U_{\text {actual }}
$$

where, $U_{\text {actual }}$ is the value collected by the device.

By formula (31), the optimal estimation prediction SOP at time $k+1$ is $P_{S O P}$.

The SOP error equation is as follows:

$$
\text { SOP error }=P_{\text {SOP }}-P_{\text {actual }}
$$

where, $P_{\text {actual }}$ is the value collected by the device. 


\subsection{SOC Initial Value of $100 \%$}

The simulation verification curve when the initial value of the SOC is $100 \%$ is shown in Figure 4.
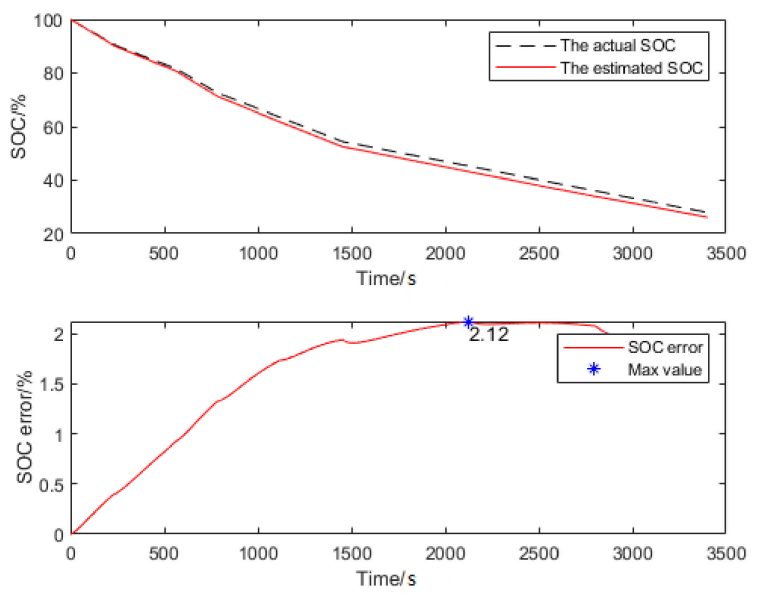

(a)
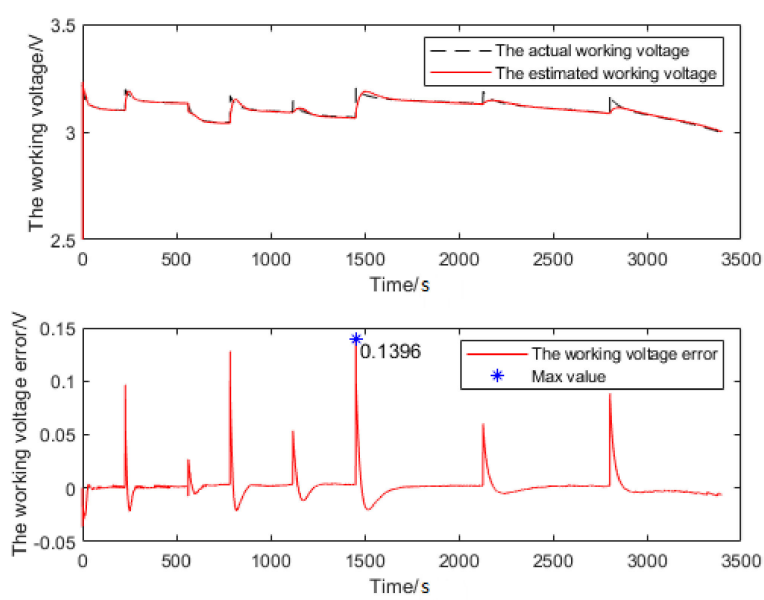

(b)
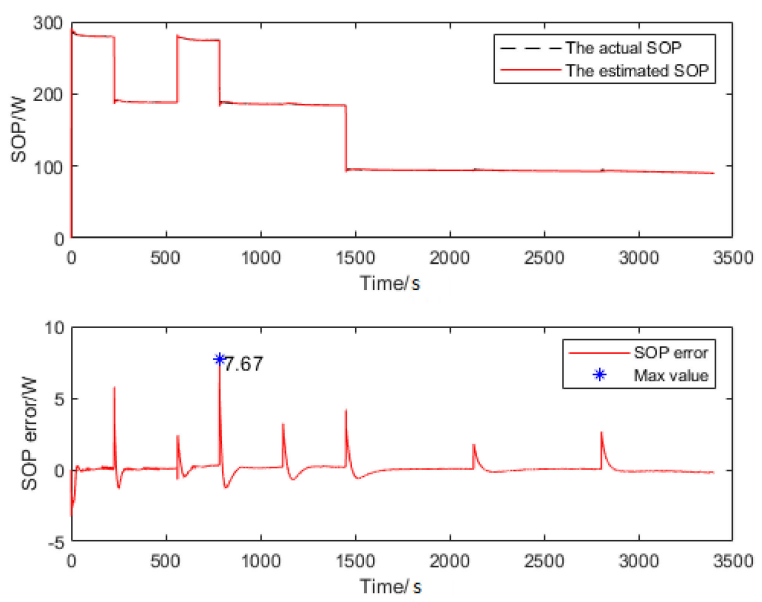

(c)

Figure 4. The simulation verification curve when the initial value of the SOC is $100 \%$. (a) The SOC estimation and error curves. (b) The working voltage estimation and error curves. (c) The SOP estimation and error curves. 
In Figure $4 \mathrm{a}$, the top graph is the adaptive curve of the echelon-use battery SOC, while the bottom graph is the error curve between the actual and the estimated values of the echelon-use battery SOC. Through a curve comparison and error analysis, the estimated error of the echelon-use battery SOC is within $2.12 \%$ (Table 2).

Table 2. Estimation error of the echelon-use battery parameters.

\begin{tabular}{cccc}
\hline $\begin{array}{c}\text { Echelon-Use Battery } \\
\text { Parameters }\end{array}$ & $\begin{array}{c}\text { Estimation Error } \\
\text { (SOC }=\mathbf{1 0 0} \%)\end{array}$ & $\begin{array}{c}\text { Estimation Error } \\
\text { (SOC }=\mathbf{7 0} \%)\end{array}$ & $\begin{array}{c}\text { Estimation Error } \\
\text { (SOC = 40\%) }\end{array}$ \\
\hline SOC & $2.12 \%$ & $-2.36 \%$ to $2.44 \%$ & $-2.49 \%$ to $2.43 \%$ \\
working voltage & $4.36 \%$ & $4.38 \%$ & $4.40 \%$ \\
SOP & $3.99 \%$ & $4.04 \%$ & $4.78 \%$ \\
\hline
\end{tabular}

In Figure $4 b$, the top graph is the adaptive curve of the echelon-use battery working voltage, while the bottom graph is the error curve between the actual and the estimated values of the echelon-use battery working voltage. Through a curve comparison and error analysis, the estimated error value is $0.1396 \mathrm{~V}$, the echelon-use battery working voltage platform is $3.2 \mathrm{~V}$, and the calculated estimated error is within $4.36 \%$ (Table 2).

In Figure 4c, the top graph is the adaptive curve of the echelon-use battery SOP, while the bottom graph is the error curve between the actual value and the estimated value of the echelon-use battery SOP. Through a curve comparison and error analysis, the estimated error value is $7.67 \mathrm{~W}$, the echelon-use battery is rated at $192 \mathrm{~W}$, and the calculated estimate error is within $3.99 \%$ (Table 2).

\subsection{SOC Initial Value of $70 \%$}

The simulation verification curve when the initial value of the SOC is $70 \%$ is shown in Figure 5.

n Figure 5a, the top graph is the adaptive curve of the echelon-use battery SOC, while the bottom graph is the error curve between the actual and the estimated values of the echelon-use battery SOC. Through a curve comparison and error analysis, the echelon-use battery SOC estimation demonstrates adaptive characteristics, and the estimated error is $-2.36 \%$ to $2.44 \%$ (Table 2 ).

In Figure $5 b$, the top graph is the adaptive curve of the echelon-use battery working voltage, while the bottom graph is the error curve between the actual and the estimated values of the echelon-use battery working voltage. Through a curve comparison and error analysis, the echelon-use battery working voltage estimation is found to exhibit adaptive characteristics. The estimated error value is $0.1401 \mathrm{~V}$, the echelon-use battery working voltage platform is $3.2 \mathrm{~V}$, and the calculated estimated error is within $4.38 \%$ (Table 2).

In Figure $5 c$, the top graph is the adaptive curve of the echelon-use battery SOP, while the bottom graph is the error curve between the actual and the estimated values of the echelon-use battery SOP. Through a curve comparison and error analysis, the echelon-use battery SOP estimation has adaptive characteristics, the estimated error value is $7.75 \mathrm{~W}$, the echelon-use battery is rated at $192 \mathrm{~W}$, and the calculated estimated error is within $4.04 \%$ (Table 2).

\subsection{SOC Initial Value of $40 \%$}

The simulation verification curve when the initial value of the SOC is $40 \%$ is shown in Figure 6.

In Figure 6a, the top graph is the adaptive curve of the echelon-use battery SOC, while the bottom graph is the error curve between the actual and the estimated values of the echelon-use battery SOC. Through a curve comparison and error analysis, the echelon-use battery SOC estimation demonstrates adaptive characteristics, and the estimated error is $-2.49 \%$ to $2.43 \%$ (Table 2 ). 

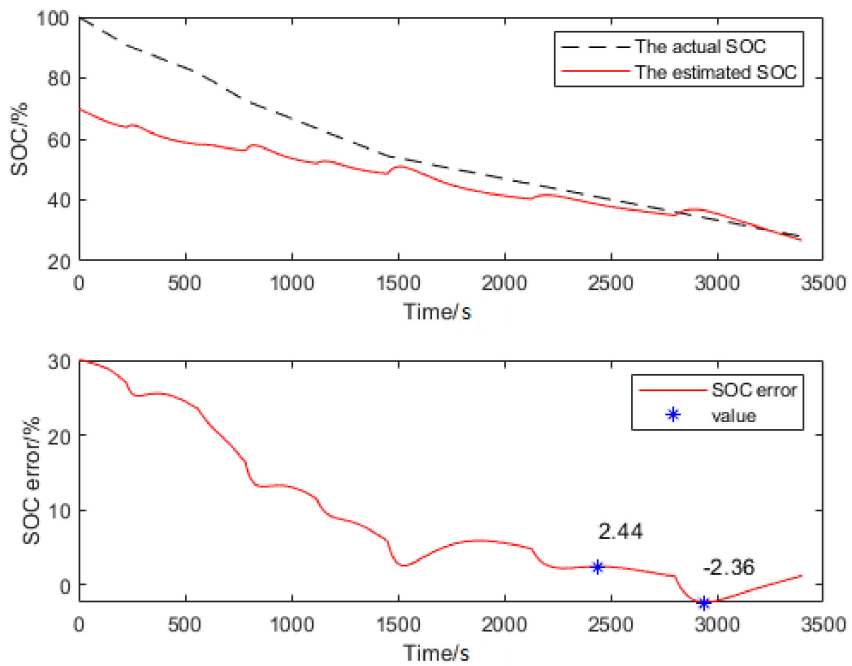

(a)
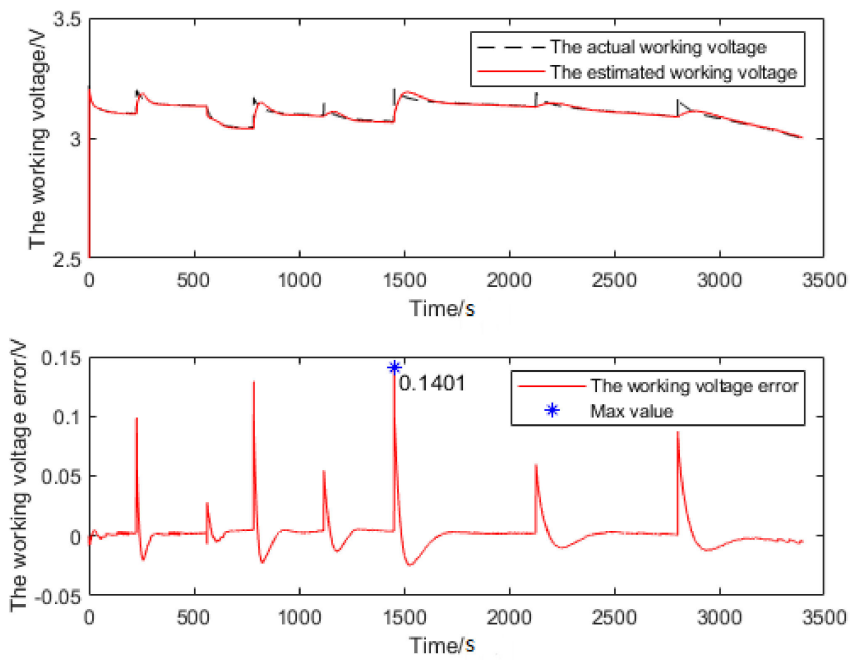

(b)
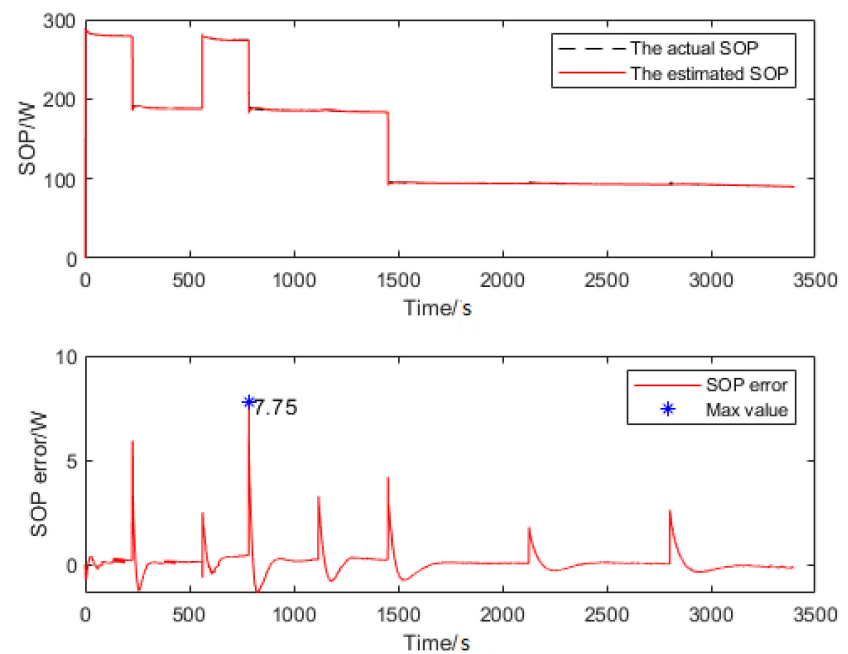

(c)

Figure 5. The simulation verification curve when the initial value of the SOC is $70 \%$. (a) The SOC estimation and error curves. (b) The working voltage estimation and error curves. (c) The SOP estimation and error curves. 

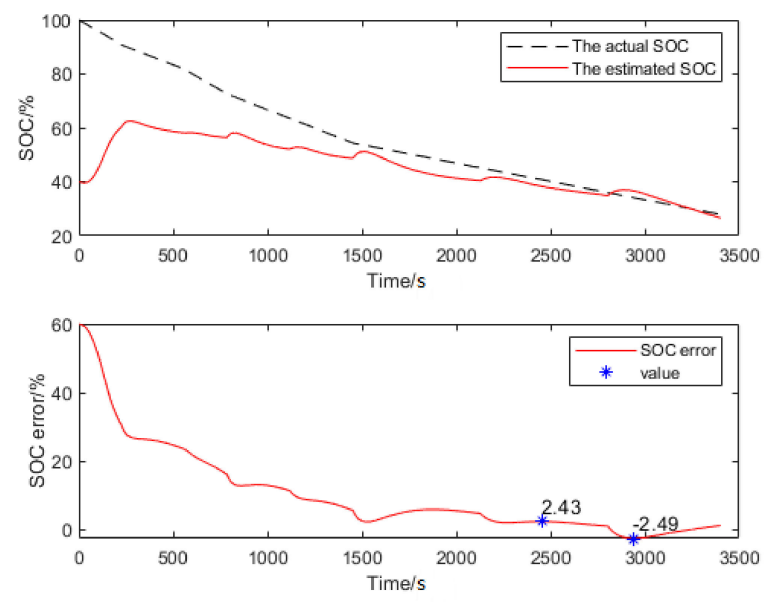

(a)
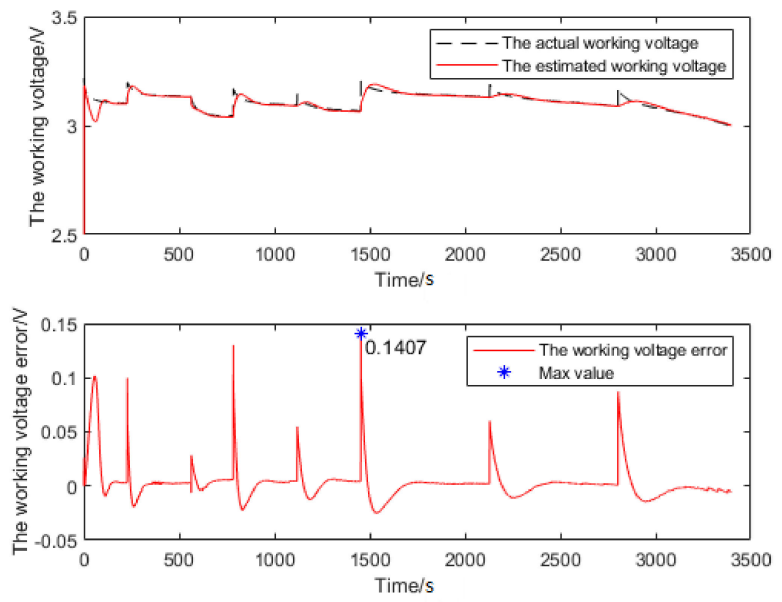

(b)
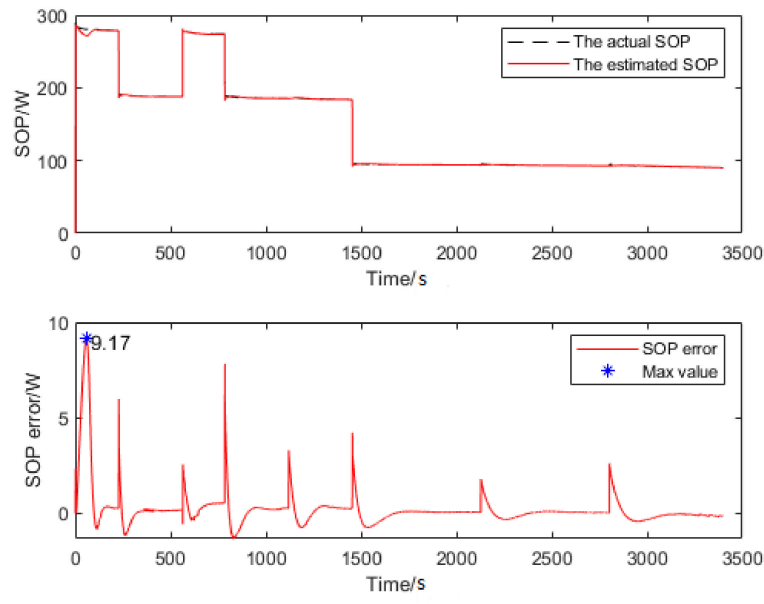

(c)

Figure 6. The simulation verification curve when the initial value of the SOC is $40 \%$. (a) The SOC estimation and error curves. (b) The working voltage estimation and error curves. (c) The SOP estimation and error curves.

In Figure $6 \mathrm{~b}$, the top graph is the adaptive curve of the echelon-use battery working voltage, while the bottom graph is the error curve between the actual and the estimated values of the echelon-use battery working voltage. Through a curve comparison and error analysis, the echelon-use battery working voltage estimation is found to exhibit adaptive 
characteristics. The estimated error value is $0.1407 \mathrm{~V}$, the echelon-use battery working voltage platform is $3.2 \mathrm{~V}$, and the calculated estimated error is within $4.40 \%$ (Table 2).

In Figure $6 c$, the top graph is the adaptive curve of the echelon-use battery SOP, while the bottom graph is the error curve between the actual and the estimated values of the echelon-use battery SOP. Through a curve comparison and error analysis, the echelon-use battery SOP estimation has adaptive characteristics, the estimated error value is $9.17 \mathrm{~W}$, the echelon-use battery is rated at $192 \mathrm{~W}$, and the calculated estimated error is within $4.78 \%$ (Table 2).

\section{Conclusions}

In this paper, the second-order Thevenin equivalent model of an echelon-use battery is established first, and then the SOP estimation method of such a battery is established by the ADEKF algorithm. A simulation analysis shows that the estimation accuracy error of the echelon-use battery SOC is less than $2.5 \%$, the estimation accuracy error of the echelon-use battery working voltage is less than $4.5 \%$, and the estimation accuracy error of the echelon-use battery SOP is less than $4.8 \%$. Therefore, the method has high accuracy.

In addition, regardless of whether or not the initial value of the SOC is clear, the proposed algorithm can be adjusted to the adaptive algorithm, and it has high accuracy.

Author Contributions: Conceptualization, Y.X. and E.H.; methodology, Y.X.; software, E.H.; validation, E.H., Y.X., and X.Q.; formal analysis, G.L.; investigation, G.L.; resources, Y.X.; data curation, E.H.; writing—original draft preparation, E.H.; writing—review and editing, Y.X.; visualization, X.Q.; supervision, Y.X.; project administration, Z.W.; funding acquisition, Y.X. All authors have read and agreed to the published version of the manuscript.

Funding: This work was supported by the Science and Technology Major Project of Inner Mongolia Autonomous Region (2020ZD0014).

Institutional Review Board Statement: Not applicable.

Informed Consent Statement: Not applicable.

Data Availability Statement: Not applicable.

Acknowledgments: This research was conducted within the University of Shandong Jiao Tong, supported by the charging-discharging equipment (BTS20-5V/4*300A/WD). We are thankful to the testing and simulation team members for their support with the experiment.

Conflicts of Interest: The authors declare no conflict of interest.

\section{References}

1. Neubauer, J.; Ahmad, P. The ability of battery second use strategies to impact plug-in electric vehicle prices and serve utility energy storage applications. J. Power Sources 2011, 196, 10351-10358. [CrossRef]

2. Tong, S.J.; Same, A.; Kootstra, M.A.; Park, J.W. Off-grid photovoltaic vehicle charge using second life lithium batteries: An experimental and numerical investigation. Appl. Energy 2013, 104, 740-750. [CrossRef]

3. Omar, N.; Daowd, M. Assessment of second life of lithium iron phosphate-based batteries. Int. Rev. Electr. Eng. (IREE) 2012, 7, 3941-3948.

4. Jiang, Y.; Jiang, J.; Zhang, C.; Zhang, W.; Gao, Y.; Li, N. State of health estimation of second- life LiFePO4 batteries for energy storage applications. J. Clean. Prod. 2018, 205, 754-762. [CrossRef]

5. Simon, F.S.; Martin, J.B.; Christian, C.; Markus, G.; Andreas, J. Correlation between capacity and impedance of lithium-ion cells during calendar and cycle life. J. Power Sources 2016, 305, 191-199. [CrossRef]

6. Zhang, Y.; Li, Y.; Tao, Y.; Ye, J.; Pan, A.; Li, X.; Liao, Q.; Wang, Z. Performance assessment of retired EV battery modules for echelon use. Energy 2020, 993, 116555. [CrossRef]

7. USABC Electric Vehicle Battery Test Procedures Manual. Revision 2; Technical Report; Office of Scientific \& Technical Information: Washington, DC, USA, 1996.

8. Wik, T.; Fridholm, B.; Kuusisto, H. Implementation and Robustness of an Analytically Based Battery State of Power. J. Power Sources 2015, 287, 448-457. [CrossRef]

9. Li, F. Test Method for Peak Output Power of Nickel Metal Hydride Power Batteries. Master's Thesis, Central South University, Changsha, China, 2007.

10. Hu, Y. Prediction Status of Peak Power of Battery on HEV. Master's Thesis, Harbin Institute of Technology, Harbin, China, 2012. 
11. Plett, G.L. High-performance Battery-pack Power Estimation using a Dynamic Cell Model. IEEE Trans. Veh. Technol. 2004, 53, 1586-1593. [CrossRef]

12. Wang, S.; Verbrugge, M.; Wang, J.S. Power Prediction from a Battery State Estimator that Incorporates Diffusion Resistance. J. Power Sources 2012, 214, 399-406. [CrossRef]

13. Wang, S.; Verbrugge, M.; Wang, J.S. Multi-parameter Battery State Estimator Based on the Adaptive and Direct Solution of the Governing Differential Equations. J. Power Sources 2011, 196, 8735-8741. [CrossRef]

14. Lin, P.; Wang, Z.; Jin, P.; Hong, J. Novel Polarization Voltage Model: Accurate Voltage and State of Power Prediction. IEEE Access 2020, 8, 92039-92049. [CrossRef]

15. Lu, J.; Chen, Z.; Yang, Y.; Lv, M. Online Estimation of State of Power for Lithium-Ion Batteries in Electric Vehicles Using Genetic Algorithm. IEEE Access 2018, 6, 20868-20880. [CrossRef]

16. Nejad, S.; Gladwin, D.T.; Stone, D.A. On-chip implementation of Extended Kalman Filter for adaptive battery states monitoring. In Proceedings of the IECON 2016-42nd Annual Conference of the IEEE Industrial Electronics Society, Florence, Italy, 23-26 October 2016; pp. 5513-5518. [CrossRef]

17. Nejad, S.; Gladwin, D.T. Online Battery State of Power Prediction Using PRBS and Extended Kalman Filter. IEEE Trans. Ind. Electron. 2020, 67, 3747-3755. [CrossRef]

18. Chen, Z.; Lu, J.; Yang, Y.; Xiong, R. Online estimation of state of power for lithium-ion battery considering the battery aging. In Proceedings of the 2017 Chinese Automation Congress (CAC), Jinan, China, 20-22 October 2017; pp. 3112-3116. [CrossRef]

19. Cheng, W.; Yi, Z.; Liang, J.; Song, Y.; Liu, D. An SOC and SOP Joint Estimation Method of Lithium-ion Batteries in Unmanned Aerial Vehicles. In Proceedings of the 2020 International Conference on Sensing, Measurement \& Data Analytics in the Era of Artificial Intelligence (ICSMD), Xi'an, China, 15-17 October 2020; pp. 247-252. [CrossRef]

20. Hou, E.; Qiao, X.; Liu, G. Modeling and Simulation of Power Lithium-ion Battery SOC. Chin. Comput. Simul. 2014, 31, 193-196.

21. Hou, E.; Qiao, X.; Liu, G.; Li, Y. Influence of temperature on parameters of power lithium-ion battery based on RC equivalent circuit. Chin. J. Power Sources 2015, 39, 287-289.

22. Cao, L.; Huang, J.; Cao, M.; Yang, K. SOC and internal resistance estimation of lithium battery based on DKF. J. Nanchang Univ. (Eng. Technol.) 2018, 40, 179-183.

23. Hou, E.; Qiao, X.; Liu, G. Estimation of power lithium-ion battery SOC based on fuzzy optimal decision. Chin. J. Power Sources 2017, 41, 920-922.

24. Shi, J.; Li, B.; Liu, M.F. Study on degenerate of lithium-ion battery based on fuzzy inference system. Chin. J. Power Sources 2018, 42, 1488-1490.

25. Gu, Y.; Zhang, Y. SOC Estimation of Lithium Battery Based on Double Kalman Filter. Chin. J. Power Sources 2016, 40, 986-989.

26. Liu, X.; He, Y.; Zeng, G.J. Power State Estimation of Lithium Battery Considering Temperature Effect. Chin. J. Power Technol. 2016, 31, 155-163.

27. She, L.Y. Research on Joint Estimation of SOC and SOP for Vehicle Power Battery. Master's Thesis, Wuhan University of Technology, Wuhan, China, 2018. 